\title{
Recent topics of infrared effective lattice QCD *
}

\section{August 1999}

\author{
Tsuneo Suzuki ${ }^{a}$, Shun-ichi Kitahara ${ }^{b}$, Fumiyoshi Shojic ${ }^{c}$, Atsushi Nakamura ${ }^{c}$, Kentarou Yamagishi ${ }^{a}$, \\ Shoichi Ito $^{\text {a }}$, Tomohiro Tsunemi ${ }^{a}$, Shouji Fujimoto ${ }^{a}$, Seikou Kato ${ }^{a}$ and Hiroaki Kodama ${ }^{a}$ \\ aDepartment of Physics, Kanazawa University, Kanazawa 920-1192, Japan \\ bJumonji University, Niiza,Saitama 352-8510, Japan \\ ${ }^{\mathrm{c}}$ Research Institute for Information Science and Education, Hiroshima University, Higashi-Hiroshima \\ 739-8521, Japan
}

Three topics concerning infrared effective lattice QCD are discussed. (1)Perfect lattice action of infrared $S U(3)$ QCD and perfect operators for the static potential are analytically given when we assume two-point monopole interactions alone. The assumption seems to be justified from numerical analyses of pure $S U(3)$ QCD in maximally abelian gauge. (2)Gauge invariance of monopole dominance can be proved theoretically if the gauge invariance of abelian dominance is proved. The gauge invariance of monopole condensation leads us to confinement of abelian neutral but color octet states after abelian projection. (3)A stochastic gauge fixing method is developed to study the gauge dependence of the Abelian projection, which interpolates between the maximally abelian (MA) gauge and no gauge fixing. Abelian dominance for the heavy quark potential holds even in the gauge which is far from Maximally Abelian one.

\section{SU3 infrared effective monopole action}

Abelian dominance and monopole dominance in MA gauge suggest the existence of an effective monopole action also in $S U(3)$ QCD. There are two independent (three with one constraint $\left.\sum_{i=1}^{3} k_{\mu}^{i}(s)=0\right)$ currents in the case of $S U(3)$. Applying the same inverse Monte-Carlo method developed in the case of $S U(2) \mathrm{QCD}[1$,2], we have derived the infrared effective monopole action starting from the vacuum ensemble $\left\{k_{\mu}^{i}(s)\right\}$ defined from the thermalized abelian link fields after the MA abelian projection[3]. Effective monopole actions can be derived similarly for the blocked monopole currents $K_{\mu}^{\alpha}(s)=\sum_{i, j, m=0}^{n-1} k_{\mu}^{\alpha}(n s+$ $(n-1) \hat{\mu}+i \hat{\nu}+j \hat{\rho}+m \hat{\sigma})[4]$. This corresponds to the block-spin transformation of the monopole currents on the dual lattice. The form of the action is restricted to 27 two-point interactions up to $3 n a(\beta)$ distance between monopole currents and the most leading 4 and 6 point interactions. The lattice size is $48^{4}$ for $\beta=5.6 \sim 6.4$. The results are as follow:

\footnotetext{
*The authors of each part are 1. K.Yamagishi, S.Kitahara and T.Suzuki, 2. T.Suzuki, S.Ito, T.Tsunemi, S.Fujimoto and S.Kato, 3. F.Shoji, T. Suzuki, H. Kodama and A. Nakamura
}

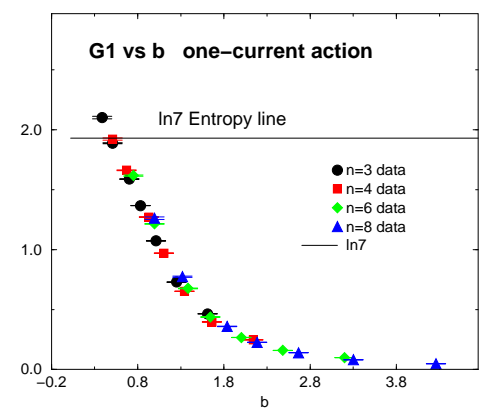

Figure 1. The self coupling in the one-current case.

1. The monopole actions are fixed clearly when we pay attention to two independent monopole currents: $S(k)=\sum_{i} G_{i}\left(S_{i}\left(k^{1}\right)+\right.$ $S_{i}\left(k^{2}\right)+S_{i}\left(-k^{1}-k^{2}\right)$ as well as one kind of monopole currents: $S(k)=\sum_{i} G_{i} S_{i}(k)$.

2. The scaling $S(n, a(\beta))=S(b=n a(\beta))$ for fixed $b=n a(\beta)$ looks good both for onecurrent and two-current cases. (Fig.1)

3. Monopole condensation occurs in the large $b$ region. Entropy dominates over the energy: $\ln 7>G_{1}$ in the one-current case. (Fig.1)

4. The quadratic monopole actions are dominant for large $b$ in both cases.

In the infrared regions, quadratic monopole interactions are dominant. When we restrict our- 
selves to quadratic monopole interactions alone, the block-spin transformation can be done analytically as done in $S U(2) \mathrm{QCD}[5]$. We can obtain also the perfect operator to evaluate the static quark-antiquark potential $V(I b, J b, K b)$ on the coarse $b$ lattice.

In the framework of the string model, the quantum effects are seen to be small in the infrared region and the static potential is evaluated by the classical contributions alone:

$$
\begin{aligned}
\langle W(C)\rangle_{c l} & =\exp \left\{-\frac{2}{3} \pi^{2} a^{8} \sum_{s, s^{\prime}, \mu, \nu} N_{\mu}(a s)\right. \\
& \left.\times A_{\mu \nu}\left(a s-a s^{\prime}\right) N_{\nu}\left(a s^{\prime}\right)\right\},
\end{aligned}
$$

where $N_{\mu}(s)=\sum \Delta^{-1}\left(s-s^{\prime}\right) \frac{1}{2} \epsilon_{\mu \alpha \beta \gamma} \partial S_{\beta \gamma}\left(s^{\prime}+\hat{\mu}\right)$ and $S_{\beta \gamma}(s)$ is the source term corresponding to the Wilson loop. We get the rotational invariance $V(I b, I b, 0) / V(I b, 0,0)=\sqrt{2}$ and $\sigma=$ $\pi \kappa \ln \left(m_{1} / m_{2}\right) / 3$, where $m_{1}$ and $m_{2}$ are dimensional constants parametrizing the monopole action on the fine $a$ lattice. $\sigma$ is equivalent to the result from DGL theory [6].

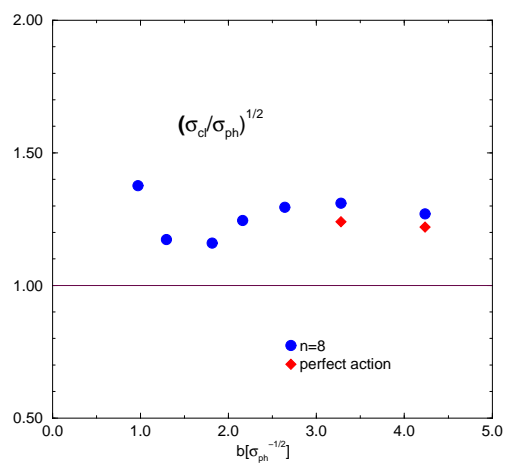

Figure 2. The string tension evaluated from the $S U(3)$ effective action.

\section{Gauge independence of abelian and monopole dominances}

Abelian and monopole dominances are shown numerically in MA gauge. However, monopole condensation after abelian projection in some gauges can not explain color confinement. Abelian neutral but color non-singlet states are not confined. Gauge independence of monopole condensation is essential for color confinement. Here we prove the gauge independence of the monopole dominance if abelian dominance is gauge independent.

First we note that abelian Wilson loops without any gauge fixing are known to give the same string tension [7,8] as that from non-abelian Wilson loops in the strong coupling expansion. Recently, Ogilivie [9] proved the same fact for any $\beta$ using the character expansion [8]. Abelian dominance comes from the Elitzur's theorem[10] and then it must be gauge independent.

Abelian dominance leads us to abelian effective action. We express the abelian action in terms of the Villain form $Z=\int_{-\pi}^{\pi} D \theta \sum_{n \in \mathbf{Z}} \mathrm{e}^{-F[d \theta+2 \pi n]}$. The general Villain action can be expressed as follows:

$$
\begin{aligned}
& \mathrm{e}^{-(d \theta+2 \pi n, D(d \theta+2 \pi n))-F^{\prime}[d \theta+2 \pi n]} \\
& =\left.e^{-F^{\prime}[-i \delta / \delta B]} \mathrm{e}^{-(d \theta+2 \pi n, D(d \theta+2 \pi n))+i(B, d \theta+2 \pi n)}\right|_{B=0},
\end{aligned}
$$

where $[D, d]=[D, \delta]=0$ are satisfied in the large $\beta$ scaling region.

The abelian Wilson loop $\mathrm{e}^{i(\theta, J)}$ is estimated with this action, where $J$ is the electric current. When we use the BKT transformation[1], we get an action in terms of monopole currents:

$$
\begin{aligned}
& Z(J)=e^{-F^{\prime}[-i \delta / \delta B]} \sum_{k \in \mathbf{Z}, d k=0} \mathrm{e}^{-\frac{1}{4}\left(\delta B,(\Delta D)^{-1} \delta B\right)} \\
& \mathrm{e}^{\frac{1}{2}\left(i B, 4 \pi \delta \Delta^{-1} k+i(\Delta D)^{-1} d J\right)} \mathrm{e}^{-4 \pi^{2}\left(k, \Delta^{-1} D k\right)} \\
& \left.\mathrm{e}^{2 \pi i\left(\delta \Delta^{-1} k, S\right)} \mathrm{e}^{-\frac{1}{4}\left(J,(\Delta D)^{-1} J\right)}\right|_{B=0}
\end{aligned}
$$

1)Electric-electric current $J-J$ interactions (with no monopole $k$ ) come from the exchange of regular photons and have no line singularity leading to a linear potential. Hence the linear potential of abelian Wilson loops is due to the monopole contribution alone. Monopole dominance is proved from abelian dominance. 2) The linear potential comes only from $\exp \left(2 \pi i\left(\delta \Delta^{-1} k, S\right)\right)$. The surface independence of the static potential is assured due to the $4-\mathrm{d}$ linking number.

\section{A new gauge fixing method for abelian projection}

To confirm the above results numerically, we analyze gauge dependence of abelian projection 
by Langevin equation with stochastic gauge fixing term[12],

$\frac{\partial}{\partial \tau} A_{\mu}^{a}(x, \tau)=-\frac{\delta S}{\delta A_{\mu}^{a}(x, \tau)}+\frac{1}{\alpha} D_{\mu}^{a b} \Delta^{b}+\eta_{\mu}^{a}(x, \tau),(1)$

where $\tau$ is fictious time, $\eta$ is Gaussian white noise and $\Delta$ is gauge fixing term. $\alpha=0(\alpha=\infty)$ corresponds to the MA gauge fixing (no gauge fixing).

A compact lattice version of Eq.(1) was proposed in Ref. 123.

$U_{\mu}(x, \tau+\Delta \tau)=\omega^{\dagger}(x, \tau) e^{i f_{\mu}^{a} t^{a}} U_{\mu}(x, \tau) \omega(x+\hat{\mu}, \tau)$,

$f_{\mu}^{a}=-\frac{\partial S}{\partial A_{\mu}^{a}} \Delta \tau+\eta_{\mu}^{a}(x, \tau) \sqrt{\Delta \tau}$,

$\omega(x, \tau)=\exp \left(i \beta \Delta^{a}(x, \tau) t^{a} \Delta \tau / 2 N_{c} \alpha\right)$.

We set $\Delta(x, \tau)=i\left[\sigma_{3}, X(x, \tau)\right]$, where $X$ is the operator to be diagonalized in MA gauge.

As a first test, we perform numerical simulations on a $12^{4}$ lattice with $\beta=2.35, \alpha=$ $0.025,0.25,2.5$, and $\Delta \tau=0.001$. We show the abelian heavy quark potentials for different $\alpha$ in Fig. 3 together with that of non-abelian potential, which are essentially same from $\alpha=0$ to 2.5 , and show the confinement linear potential behavior.

Note that the string tension obtained by the standard gauge fixing procedure is larger than that of $\alpha=0$. This is probably due to the Gribov copies 14. Bali et al. [15] have observed that when the number of copies decreases the string tension becomes small. This is very consistent with our data.

The heavy quark potentials from monopole and photon contributions are plotted in Fig.4. The monopole part shows the confinement behavior.

T.S. acknowledges financial support from JSPS Grant-in Aid for Scientific Research (B) (No.10440073 and No.11695029).

\section{REFERENCES}

1. H.Shiba and T.Suzuki, Phys. Lett. B351(1995) 519.

2. S.Kato et al., Nucl. Phys. B520(1998) 323.

3. T.A. DeGrand and D. Toussaint, Phys. Rev. D22 (1980) 2478.

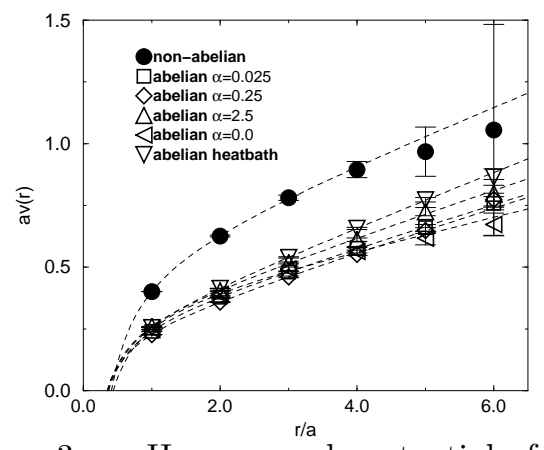

Figure 3. Heavy quark potentials from nonabelian and abelian contributions.

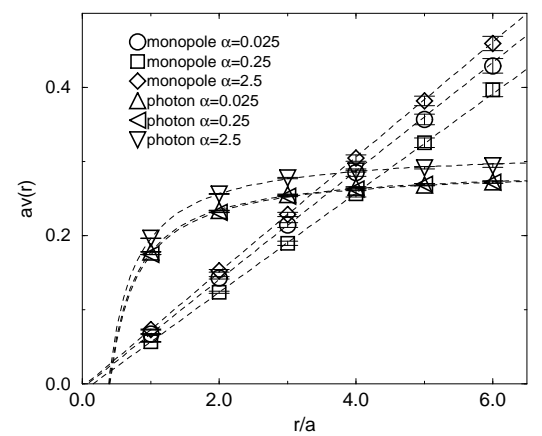

Figure 4. Heavy quark potentials from monopole and photon contributions.

4. T.L. Ivanenko et al., Phys. Lett. 252B (1990) 631.

5. S.Fujimoto et al., in this proceedings.

6. T. Suzuki, Prog.Theor.Phys. 80 (1988) 929;

S. Maedan and T. Suzuki, Prog.Theor.Phys. 81 (1988) 229.

7. T. Suzuki and I. Yotsuyanagi, Phys. Rev. D42 (1990) 4257

8. M.Faber et al., hep-lat/9810008.

9. M.C. Ogilvie,Phys.Rev. D59 (1999) 074505.

10. S.Elitzur, Phys. Rev. D12 (1975) 3978.

11. V.L. Beresinskii, Sov. Phys. JETP 32 (1970) 493; J.M. Kosterlitz and D.J. Thouless, J. Phys.,C6 (1973) 1181.

12. D.Zwanziger, Nucl. Phys. B192 (1981) 259.

13. M.Mizutani and A.Nakamura, Vistas in Astronomy, 37, 305, 1993, Pergamon Press; Nucl.Phys. B(Proc.Suppl.)34, 253, 1994.

14. S.Hioki et al., Phys.Lett. B272 (1991) 326.

15. G.S.Bali et al.,Phys.Rev. D54 (1996) 2863. 